\title{
Balance Laws and Weak Boundary Conditions in Continuum Mechanics
}

\author{
ALFREDO MARZOCCHI ${ }^{1}$ and ALESSANDRO MUSESTI ${ }^{2, \star}$ \\ ${ }_{1}^{1}$ Dipartimento di Matematica, Università di Brescia, Via Valotti 9, I-25133 Brescia, Italy. \\ E-mail:amarzocc@ing.unibs.it \\ ${ }^{2}$ Dipartimento di Matematica e Fisica, Università Cattolica del Sacro Cuore, Via dei Musei 41, \\ I-25121 Brescia, Italy.E-mail: a.musesti@dmf.unicatt.it
}

\section{Dedicated to Carlo Banfi on the occasion of his 70th birthday}

Received 8 October 2003; in revised form 13 May 2004

Abstract. A weak formulation of the stress boundary conditions in Continuum Mechanics is proposed. This condition has the form of a balance law, allows also singular measure data and is consistent with the regular case. An application to the Flamant solution in linear elasticity is shown.

Mathematics Subject Classifications (2000): 74A10, 74G70.

Key words: boundary conditions, stress, balance laws.

\section{Introduction}

In this note we aim at extending the notion of assigning the applied traction on the boundary of a continuous body to cases where the given stress field lacks regularity (it can even be not pointwise defined), but obeys some measure-theoretic laws as additivity with respect to pieces of the boundary.

The key tool here is the concept of balance law. In the interior of the body it is well-known that, in the classical case, the momentum balance laws imply the existence and the symmetry of the Cauchy stress tensor field $\mathrm{T}$; this can be obtained, for instance, via the tetrahedron argument. When this procedure is applied to the boundary of the body, the normal is fixed and "linearity in the normal" does not make sense. However, another result is achieved: whenever the normal component $\mathrm{Tn}$ of the stress tensor field admits a trace on the boundary of the body, then the balance implies that an assigned boundary datum must equal this trace value (like, for instance, in boundary-value problems). So the balance law can be viewed as another way to assign the stress vector field on the boundary, possibly

\footnotetext{
$\star$ The authors thank Paolo Podio-Guidugli for a careful revision of the paper.

The research of the authors was partially supported by the MIUR project "Modelli matematici per la scienza dei materiali" (COFIN 2002) and by Gruppo Nazionale per la Fisica Matematica (INdAM).
} 
including the case of singular loads. This is in agreement with the usual point of view of continuum mechanics, which interprets singular solutions in elastostatics as guaranteeing the force balance of subbodies containing the singular set.

In non-classical situations, that is, when the regularity of the stress tensor field is not guaranteed, many results have been given in the recent past $[7,4,3,10$, 11]. In particular, in [2] the stress field is a $L_{\text {loc }}^{1}$-function with divergence measure and the associated flux makes sense only for almost every material surface (in a suitable sense, see also [11]). However, these results do not hold on the boundary of the body: specifically, the fact that $\mathbf{T n}$ have a trace on the boundary is not a consequence of the balance law (nor it is expected to be, as in the distributional approach the balance laws are typically formulated for inner subbodies).

Therefore, we seek to modify the notion of balance law in order to formulate it up to the boundary and we propose it as a weak form of formulating the traction boundary conditions. By means of mathematical tools borrowed from Geometric Measure Theory and already used in [2, 6], we prove that our approach is consistent with the classical pillbox argument. More important, the proposed traction boundary condition (BC) is such that the corresponding flux cannot have distinct extensions to the boundary from the interior of the body, as is natural (Theorem 4.1). We show that, under weak assumptions on the stress field given at the boundary, it is now possible to assign a Cauchy flux at the boundary, which corresponds to a vector-valued Radon measure. Moreover, an approximation process from the interior of the body can be performed in order to obtain the value of the boundary datum (Theorem 4.2). Finally, as an example we revisit the classical Flamant solution of a concentrated load at the boundary of a linearly elastic half-plane, showing that our theory covers also this case. Of course, for a thermodynamical viewpoint one can decrease the tensorial order and replace "stress" by "heat".

It is worth noting that we do not deal with boundary-value problems, but only with the setting of the stress boundary conditions. A weakening of this concept can be useful, for instance, when boundary data involve singular loads, as pointed out in [9]. Moreover, we do not cover the case of mixed boundary conditions, because our result does not depend on constitutive laws and the displacements do not come into play. On the other hand, the balance condition we propose, besides its mechanical interest, can be regarded from a strictly mathematical point of view as a sufficient condition for the existence of the trace at the boundary (at least in a weak sense) of the normal component of a tensor field.

In [2] it was proved that a balance law can be postulated without loss of generality only on almost every $n$-interval. A measure-theoretic argument then shows that that balance law holds true for almost every subbody with finite perimeter, that is to say, for a very large class of subbodies (see [1, 8]). For this reason, we have chosen to state the balance law (or, equivalently, to assign the Cauchy flux) on figures, which are finite unions of $n$-intervals. Anyway, since here the boundary of the body is given, it is no more clear whether the balance condition holds for all subbodies with finite perimeter up to the boundary; the question is still open. 


\section{Background Material}

For $n \geqslant 1, \mathscr{L}^{n}$ will denote $n$-dimensional Lebesgue outer measure, and $\mathscr{H}^{k}$ $k$-dimensional Hausdorff outer measure on $\mathbb{R}^{n}$. Given a Borel subset $E \subseteq \mathbb{R}^{n}$, we denote by $\mathfrak{B}(E)$ the collection of all Borel subsets of $E$, that is, the $\sigma$-algebra generated by the open subsets of $E$. Moreover, $E \triangle F$ will denote the set $(E \backslash F) \cup$ $(F \backslash E)$.

Consider a set $M \subseteq \mathbb{R}^{n}$. The topological interior, closure and boundary of $M$ will be denoted by int $M, \operatorname{cl} M$ and bd $M$, respectively. Denoting by $B_{r}(x)$ the open ball with radius $r$ centered at $x$, we introduce the measure-theoretic interior of $M$

$$
M_{*}=\left\{x \in \mathbb{R}^{n}: \lim _{r \rightarrow 0^{+}}\left[r^{-n} \mathscr{L}^{n}\left(B_{r}(x) \backslash M\right)\right]=0\right\},
$$

the measure-theoretic closure of $M$

$$
M^{*}=\mathbb{R}^{n} \backslash\left(\mathbb{R}^{n} \backslash M\right)_{*}
$$

and the measure-theoretic boundary of $M$

$$
\partial_{*} M=M^{*} \cap\left(\mathbb{R}^{n} \backslash M\right)^{*} .
$$

It is easy to see that $\partial_{*} M=\mathbb{R}^{n} \backslash\left(M_{*} \cup\left(\mathbb{R}^{n} \backslash M\right)_{*}\right)$. Moreover, since $M_{*} \cap\left(\mathbb{R}^{n} \backslash M\right)_{*}=$ $\emptyset$, one has $M^{*}=M_{*} \cup \partial_{*} M$.

REMARK 2.1. If $M$ has a locally Lipschitz boundary, then $\partial_{*} M=\mathrm{bd} M$.

PROPOSITION 2.2. Let $M \subseteq \mathbb{R}^{n}$. Then the following properties hold:

(a) int $M \subseteq M_{*} \subseteq M^{*} \subseteq \mathrm{cl} M$;

(b) $M_{*}, M^{*}, \partial_{*} M \in \mathfrak{B}\left(\mathbb{R}^{n}\right)$;

(c) $\mathscr{L}^{n}\left(M_{*} \backslash M\right)=0$; moreover, $M$ is $\mathscr{L}^{n}$-measurable if and only if $\mathcal{L}^{n}\left(M \backslash M_{*}\right)=0$

(d) if $M \subseteq N$, then $M_{*} \subseteq N_{*}$ and $M^{*} \subseteq N^{*}$.

Proof. (a) is obvious, while (b) can be proved by remarking that

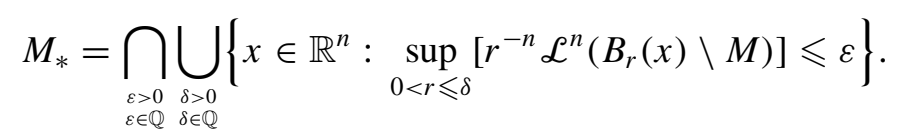

Property (c) follows by standard arguments of Measure Theory. Let us prove (d). One has $B_{r}(x) \backslash N \subseteq B_{r}(x) \backslash M$; if $x \in M_{*}$ it follows that $r^{-n} \mathcal{L}^{n}\left(B_{r}(x) \backslash N\right) \rightarrow 0$ as $r \rightarrow 0$, hence $x \in N_{*}$. Moreover, $\left(\mathbb{R}^{n} \backslash N\right)_{*} \subseteq\left(\mathbb{R}^{n} \backslash M\right)_{*}$ and the proof is complete.

Let $M \subseteq \mathbb{R}^{n}$ and $x \in \partial_{*} M$. Let $\mathcal{V}$ be the linear space associated to $\mathbb{R}^{n}$. We denote by $\boldsymbol{n}^{M}(x) \in \mathcal{V}$ a unit vector such that

$$
\begin{aligned}
& \lim _{r \rightarrow 0^{+}}\left[r^{-n} \mathscr{L}^{n}\left(\left\{\xi \in B_{r}(x) \cap M:(\xi-x) \cdot \boldsymbol{n}^{M}(x)>0\right\}\right)\right]=0, \\
& \lim _{r \rightarrow 0^{+}}\left[r^{-n} \mathscr{L}^{n}\left(\left\{\xi \in B_{r}(x) \backslash M:(\xi-x) \cdot \boldsymbol{n}^{M}(x)<0\right\}\right)\right]=0 .
\end{aligned}
$$


No more than one such vector can exist. When the limits do not both obtain, we set $\boldsymbol{n}^{M}(x)=\mathbf{0}$. The bounded map $\boldsymbol{n}^{M}: \partial_{*} M \rightarrow \mathcal{V}$ is called the unit outer normal to $M$. It turns out that $\boldsymbol{n}^{M}$ is a Borel map, that is, $\left(\boldsymbol{n}^{M}\right)^{-1}(A) \in \mathfrak{B}\left(\partial_{*} M\right)$ for any open subset $A \subseteq \mathbb{R}^{n}$.

DEFINITION 2.3. Let $M \subseteq \mathbb{R}^{n}$. We say that $M$ is a set with finite perimeter, if $\mathscr{H}^{n-1}\left(\partial_{*} M\right)<+\infty$.

The choice of sets with finite perimeter as subbodies is due to the fact that the unit outer normal exists $\mathscr{H}^{n-1}$-a.e. on the measure-theoretic boundary and the divergence theorem holds.

PROPOSITION 2.4. Let $M, N$ be two $\mathcal{L}^{n}$-measurable subsets of $\mathbb{R}^{n}$ of finite perimeter and let $A=\left(\partial_{*} M \backslash\left(N_{*} \cup \partial_{*} N\right)\right), B=\left(\partial_{*} N \backslash\left(M_{*} \cup \partial_{*} M\right)\right)$, $C=\left(M_{*} \cap \partial_{*} N\right), D=\left(N_{*} \cap \partial_{*} M\right)$,

$$
\begin{aligned}
& E=\left\{x \in \partial_{*} M \cap \partial_{*} N: \boldsymbol{n}^{M}(x) \neq 0, \boldsymbol{n}^{N}(x) \neq 0, \boldsymbol{n}^{M}(x) \neq-\boldsymbol{n}^{N}(x)\right\}, \\
& F=\left\{x \in \partial_{*} M \cap \partial_{*} N: \boldsymbol{n}^{M}(x) \neq 0, \boldsymbol{n}^{N}(x) \neq 0, \boldsymbol{n}^{M}(x) \neq \boldsymbol{n}^{N}(x)\right\} .
\end{aligned}
$$

Then there exist three sets $R_{k} \subseteq \partial_{*} M \cap \partial_{*} N$, for $k=1,2,3$, such that $\mathscr{H}^{n-1}\left(R_{k}\right)=0$ and

$$
\begin{aligned}
& \partial_{*}(M \cup N)=A \cup B \cup E \cup R_{1}, \\
& \partial_{*}(M \cap N)=C \cup D \cup E \cup R_{2}, \\
& \partial_{*}(M \backslash N)=A \cup C \cup F \cup R_{3},
\end{aligned}
$$

where the unions are disjoint.

See [6, Proposition 2.2].

\section{The Cauchy Flux}

Let $B$ be an open, bounded, normalized subset of $\mathbb{R}^{n}$ with finite perimeter and set

$$
\begin{aligned}
& \mathcal{M}=\left\{M \in \mathfrak{B}(B): M=M_{*}, \mathscr{H}^{n-1}\left(\partial_{*} M\right)<+\infty\right\}, \\
& \mathcal{M}^{\circ}=\{M \in \mathcal{M}: \operatorname{cl} M \subseteq B\} .
\end{aligned}
$$

The elements of $\mathcal{M}^{\circ}$ cannot share any part of their boundary with the boundary of the whole body $B$.

DEFINITION 3.1. A material surface is a pair $S=\left(\widehat{S}, \boldsymbol{n}_{S}\right)$, where $\widehat{S}$ is a Borel subset of $B$ and $\boldsymbol{n}_{S}: \widehat{S} \rightarrow \mathcal{V}$ is a Borel map such that there exists $M \in \mathcal{M}$ with $\widehat{S} \subseteq \partial_{*} M$ and $\boldsymbol{n}_{S}=\left.\boldsymbol{n}^{M}\right|_{\widehat{S}}$. In this case, we say that $S$ is subordinate to $M$. We denote by $\mathcal{S}$ the collection of material surfaces, and by $\mathcal{S}^{\circ}$ the collection of material surfaces subordinated to some $M \in \mathcal{M}^{\circ}$.

We call $\boldsymbol{n}_{S}$ the normal to the surface $S$. Two material surfaces $S$ and $T$ are said to be compatible, if they both are subordinate to the same $M$. 
Note that, in any case, pieces of the measure-theoretic boundary of $B$ are not regarded as to be material surfaces. The physical intuition suggests us that the boundary of the whole body is of a different nature with respect to the boundary of the subbodies.

We denote by $\mathfrak{M}(B)$ the collection of all Borel measures $\mu: \mathfrak{B}(B) \rightarrow[0,+\infty)$ with $\mu(B)<+\infty$ and by $\mathcal{L}_{+}^{1}(B)$ the set of all Borel functions $h: B \rightarrow[0,+\infty]$ with $\int_{B} h \mathrm{~d} \mathscr{L}^{n}<+\infty$.

DEFINITION 3.2. Given $h \in \mathcal{L}_{+}^{1}(B)$ and $\eta \in \mathfrak{M}(B)$, we set

$$
\begin{aligned}
& \mathcal{M}_{h \eta}=\left\{A \in \mathcal{M}: \int_{B \cap \partial_{*} A} h \mathrm{~d} \mathcal{H}^{n-1}<+\infty, \eta\left(B \cap \partial_{*} A\right)=0\right\}, \\
& \mathcal{S}_{h \eta}=\left\{S \in \mathcal{S}: S \text { is subordinate to some } A \in \mathcal{M}_{h \eta}\right\} .
\end{aligned}
$$

We will say that a property $\pi$ holds on almost all of $\mathcal{M}$ (resp. $\mathcal{S}$ ), if there are $h \in \mathcal{L}_{+}^{1}(B)$ and $\eta \in \mathfrak{M}(B)$ such that $\pi$ holds on $\mathcal{M}_{h \eta}$ (resp. $\left.\mathcal{S}\right)$.

REMARK 3.3. It can be proved that if $h \in \mathcal{L}_{+}^{1}(B), \eta \in \mathfrak{M}(B)$ and $M_{1}, M_{2} \in$ $\mathcal{M}_{h \eta}$, then $\left(M_{1} \cup M_{2}\right)_{*}, M_{1} \cap M_{2},\left(M_{1} \backslash M_{2}\right)_{*} \in \mathcal{M}_{h \eta}$.

A similar definition can be stated for $\mathcal{M}^{\circ}$ and $\mathcal{S}^{\circ}$.

DEFINITION 3.4. Given $h \in \mathcal{L}_{+}^{1}(B)$ and $\eta \in \mathfrak{M}(B)$, we set

$$
\begin{aligned}
& \mathcal{M}_{h \eta}^{\circ}=\left\{A \in \mathcal{M}: \int_{\partial_{*} A} h \mathrm{~d} \mathscr{H}^{n-1}<+\infty, \eta\left(\partial_{*} A\right)=0\right\}, \\
& \mathcal{S}_{h \eta}^{\circ}=\left\{S \in \mathcal{S}: S \text { is subordinate to some } A \in \mathcal{M}_{h \eta}^{\circ}\right\} .
\end{aligned}
$$

We will say that a property $\pi$ holds on almost all of $\mathcal{M}^{\circ}$ (resp. $\mathcal{S}^{\circ}$ ), if there are $h \in \mathcal{L}_{+}^{1}(B)$ and $\eta \in \mathfrak{M}(B)$ such that $\pi$ holds on $\mathcal{M}_{h \eta}^{\circ}\left(\right.$ resp. $\left.\mathcal{S}^{\circ}\right)$.

DEFINITION 3.5. A grid $G$ is an ordered triple

$$
G=\left(x_{0},\left(e_{1}, \ldots, e_{n}\right), \widehat{G}\right),
$$

where $x_{0} \in \mathbb{R}^{n},\left(e_{1}, \ldots, e_{n}\right)$ is a positively oriented orthonormal basis in $\mathbb{R}^{n}$ and $\widehat{G}$ is a Borel subset of $\mathbb{R}$. If $G_{1}, G_{2}$ are two grids, we write $G_{1} \subseteq G_{2}$ if $\widehat{G}_{1} \subseteq \widehat{G}_{2}$ and they share the point $x_{0}$ and the list $\left(e_{1}, \ldots, e_{n}\right)$. A grid $G$ is said to be full, if $\mathcal{L}^{1}(\mathbb{R} \backslash \widehat{G})=0$.

Let $G$ be a grid; a set $I \subseteq \mathbb{R}^{n}$ is said to be $a G$-interval, if

$$
I=\left\{x \in \mathbb{R}^{n}: a^{(j)}<\left(x-x_{0}\right) \cdot e_{j}<b^{(j)} \forall j=1, \ldots, n\right\}
$$

for some $a^{(1)}, b^{(1)}, \ldots, a^{(n)}, b^{(n)} \in \widehat{G}$.

A subset $M$ of $\mathbb{R}^{n}$ is said to be a G-figure, if $M=\left(\bigcup_{I \in \mathcal{F}} I\right)_{*}$, where $\mathcal{F}$ is a finite family of $G$-intervals. We set

$$
\mathcal{M}_{G}=\{M \cap B: M \text { is a } G \text {-figure }\} .
$$


The following is an easy modification of [2, Proposition 4.5].

PROPOSITION 3.6. Let $x_{0} \in \mathbb{R}^{n}$ and $\left(e_{1}, \ldots, e_{n}\right)$ be a positively oriented orthonormal basis in $\mathbb{R}^{n}$. Then for every $h \in \mathcal{L}_{+}^{1}(B)$ and $\eta \in \mathfrak{M}(B)$ there exists a full grid $G$ of the form $G=\left(x_{0},\left(e_{1}, \ldots, e_{n}\right), \widehat{G}\right)$ such that $\mathcal{M}_{G} \subseteq \mathcal{M}_{h \eta}$.

DEFINITION 3.7. Let $\mathcal{P}$ be a set containing almost all of $\mathcal{S}^{\circ}$ and consider a function $Q: \mathcal{P} \rightarrow \mathbb{R}^{N}$. We say that $Q$ is a balanced Cauchy flux on $B$, if the following properties hold:

(a) if $S, T \in \mathcal{P}$ are compatible and disjoint and $S \cup T \in \mathcal{P}$, then

$$
Q(S \cup T)=Q(S)+Q(T) ;
$$

(b) there exists $h \in \mathcal{L}_{+}^{1}(B)$ such that

$$
|Q(S)| \leqslant \int_{S} h \mathrm{~d} \mathscr{H}^{n-1}
$$

for almost every $S \in \mathcal{S}^{\circ}$;

(c) there exists $\lambda \in \mathfrak{M}(B)$ such that

$$
\left|Q\left(\partial_{*} M\right)\right| \leqslant \lambda(M)
$$

for almost every $M \in \mathcal{M}^{\circ}$.

REMARK 3.8. Usually one has $N=n$ or $N=1$.

An important result about balanced Cauchy fluxes, which is proved in [2, Theorem 7.1], is the following integral representation.

THEOREM 3.9. Let $Q$ be a balanced Cauchy flux. Then there exists an essentially unique tensor field $\mathrm{T} \in L_{\mathrm{loc}}^{1}\left(B ; \operatorname{Lin}\left(\mathbb{R}^{n} ; \mathbb{R}^{N}\right)\right)$ with divergence measure such that

$$
Q(S)=\int_{S} \operatorname{T} n_{S} \mathrm{~d} \mathscr{H}^{n-1}
$$

on almost all of $\mathcal{S}^{\circ}$.

In particular, in view of (b) of Definition 3.7 one can extend the domain of $Q$ to almost all of $\mathcal{S}$, by setting

$$
Q(S)=\int_{S} \mathrm{~T} \boldsymbol{n}_{S} \mathrm{~d} \mathscr{H}^{n-1}
$$

\section{The Balance Law for Boundary Conditions}

Let $\mu \in \mathfrak{M}\left(\partial_{*} B\right)$ and $\boldsymbol{t}_{0}: \partial_{*} B \rightarrow \mathbb{R}^{N}$ be bounded and Borel. Set

$$
\forall S \in \mathfrak{B}\left(\partial_{*} B\right): \quad Q_{0}(S):=\int_{S} \boldsymbol{t}_{0} \mathrm{~d} \mu .
$$

$Q_{0}$ will denote the boundary datum for the Cauchy flux; it means that $Q_{0}$ represents the outgoing flux of the quantity (stress, heat) through $S$. 
We formulate the boundary condition as the following assumption:

(BC) there exist $v \in \mathfrak{M}(B)$ and a full grid $G$ such that

$$
\forall M \in \mathcal{M}_{G}: \quad\left|Q\left(B \cap \partial_{*} M\right)+Q_{0}\left(\partial_{*} B \cap \partial_{*} M\right)\right| \leqslant v(M) .
$$

The previous assumption is a balance requirement, since $\left(B \cap \partial_{*} M\right) \cup$ $\left(\partial_{*} B \cap \partial_{*} M\right)=\partial_{*} M$. Whenever $\operatorname{cl} M \subseteq B$, i.e. the subbody is far from $\partial_{*} B$, then (BC) reduces to property (c) of Definition 3.7.

In the following theorem, we show that assumption (BC) is well posed, in the sense that there cannot be two distinct boundary data which correspond to the same Cauchy flux $Q$.

THEOREM 4.1. Let $G$ be a full grid and $Q_{0}, Q_{1}: \mathfrak{B}\left(\partial_{*} B\right) \rightarrow \mathbb{R}^{N}$ be two boundary data satisfying $(\mathrm{BC})$ for some $v_{0}, v_{1} \in \mathfrak{M}(B)$. Then $Q_{0}=Q_{1}$.

Proof. We first prove that, if there exist $v \in \mathfrak{M}(B)$ and a full grid $G$ such that

$$
\forall M \in \mathcal{M}_{G}: \quad\left|Q_{0}\left(\partial_{*} B \cap \partial_{*} M\right)\right| \leqslant v(M),
$$

then $Q_{0}=0$. Let $h \in \mathcal{L}_{+}^{1}(B)$ and $\eta \in \mathfrak{M}(B)$ be such that (1) holds on $\mathcal{M}_{h \eta}$. Let $G_{0}$ be a full grid such that $G_{0} \subseteq G$ and $\mathcal{M}_{G} \subseteq \mathcal{M}_{h \eta}$ (cf. Proposition 3.6). Let $S$ be a Borel subset of $\partial_{*} B$ and $\mu$ be as in (2); since $\mu(S)<+\infty$, there exists an increasing sequence $\left(K_{h}\right)$ of compact sets such that $K_{h} \subseteq S$ and $\mu\left(S \backslash K_{h}\right)<1 / h$ for every $h \geqslant 1$. Fix now $h \geqslant 1$. Let $\left(P_{m}\right)$ be a decreasing sequence of $G$-figures in $\mathbb{R}^{n}$ with $K_{h} \subseteq P_{m}$ and

$$
K_{h}=\bigcap_{m \in \mathbb{N}} \mathrm{cl} P_{m} .
$$

Then there exists an index $m_{h}$ such that $\mu\left(\left(\operatorname{cl} P_{m_{h}} \cap \partial_{*} B\right) \backslash K_{h}\right)<1 / h$. We set

$$
\forall h \geqslant 1: \quad M_{h}=P_{m_{h}} \cap B ;
$$

clearly $M_{h} \in \mathcal{M}_{G}$. Moreover, taking into account Propositions 2.2 and 2.4, it can be proved that

$$
\begin{aligned}
& \left(\partial_{*} M_{h} \cap \partial_{*} B\right) \backslash S \subseteq\left(\operatorname{cl} P_{m_{h}} \cap \partial_{*} B\right) \backslash S \subseteq\left(\operatorname{cl} P_{m_{h}} \cap \partial_{*} B\right) \backslash K_{h}, \\
& S \backslash\left(\partial_{*} M_{h} \cap \partial_{*} B\right) \subseteq S \backslash K_{h},
\end{aligned}
$$

for any $h \geqslant 1$. In particular, $\lim _{h} \mu\left(S \triangle\left(\partial_{*} M_{h} \cap \partial_{*} B\right)\right)=0$, hence

$$
\lim _{h} Q_{0}\left(\partial_{*} M_{h} \cap \partial_{*} B\right)=Q_{0}(S) .
$$

Since $\lim _{h} v\left(M_{h}\right)=0$, by (BC) we get $Q_{0}(S)=0$.

Now consider the boundary datum $Q_{0}-Q_{1}$ and let $M \in \mathcal{M}_{G}$; we have

$$
\begin{aligned}
& \left|\left(Q_{0}-Q_{1}\right)\left(\partial_{*} B \cap \partial_{*} M\right)\right| \\
& \quad \leqslant\left|Q\left(B \cap \partial_{*} M\right)+Q_{0}\left(\partial_{*} B \cap \partial_{*} M\right)\right|+\left|Q\left(B \cap \partial_{*} M\right)+Q_{1}\left(\partial_{*} B \cap \partial_{*} M\right)\right| \\
& \quad \leqslant\left(v_{0}+v_{1}\right)(M) .
\end{aligned}
$$

By the previous step it follows that $Q_{0}-Q_{1}=0$. 
We want to show now that, assuming (BC), an approximation process can be performed in the interior of the body in order to obtain the value of $Q_{0}$ on a boundary surface.

THEOREM 4.2. Assume (BC) and let $S$ be a compact subset of $\partial_{*} B$ and $\left(M_{h}\right)$ a decreasing sequence in $\mathcal{M}_{G}$ such that $S=\bigcap_{h \in \mathbb{N}} \mathrm{cl} M_{h}$. Then

$$
\lim _{h \rightarrow \infty} Q\left(B \cap \partial_{*} M_{h}\right)=-Q_{0}(S) .
$$

Proof. We set $R_{h}=\left(\partial_{*} B \cap \partial_{*} M_{h}\right) \backslash S$. Then $R_{h} \subseteq\left(\partial_{*} B \cap \operatorname{cl} M_{h}\right) \backslash S$, which is a decreasing sequence of Borel sets with empty intersection. In particular,

$$
\left|Q_{0}\left(R_{h}\right)\right| \leqslant \int_{\left(\partial_{*} B \cap \mathrm{cl} M_{h}\right) \backslash S}\left|\boldsymbol{t}_{0}\right| \mathrm{d} \mu \rightarrow 0
$$

as $h \rightarrow \infty$. On the other hand, by (BC) one gets

$$
\left|Q\left(B \cap \partial_{*} M_{h}\right)+Q_{0}(S)+Q_{0}\left(R_{h}\right)\right| \leqslant v\left(M_{h}\right) .
$$

Since the sequence $\left(M_{h}\right)$ has empty intersection, then $\lim _{h} v\left(M_{h}\right)=0$, and the proof is complete.

\section{The Regular Case}

Suppose now that $B$ has a Lipschitz boundary, that $\mathrm{T}: \operatorname{cl} B \rightarrow \operatorname{Lin}\left(\mathbb{R}^{n} ; \mathbb{R}^{N}\right)$ is Lipschitz and that the boundary datum is absolutely continuous with respect to the area measure, i.e.

$$
Q_{0}(S)=\int_{S} \boldsymbol{t}_{0} \mathrm{~d} \mathscr{H}^{n-1} .
$$

We prove the following theorem.

THEOREM 5.1. Assume (BC). Then the usual boundary condition

$$
\mathrm{T}(x) \boldsymbol{n}^{B}(x)=\boldsymbol{t}_{0}(x)
$$

holds for any $x \in$ bd $B$.

Proof. Let $G$ be a full grid as in assumption (BC) and let $M \in \mathcal{M}_{G}$. By the classical divergence theorem we get

$$
\begin{aligned}
& \left|\int_{B \cap \mathrm{bd} M} \operatorname{Tn}^{M} \mathrm{~d} \mathscr{H}^{n-1}+\int_{\mathrm{bd} B \cap \mathrm{bd} M} \operatorname{Tn}^{B} \mathrm{~d} \mathscr{H}^{n-1}\right| \\
& \quad=\left|\int_{\mathrm{bd} M} \boldsymbol{T}^{M} \mathrm{~d} \mathscr{H}^{n-1}\right|=\left|\int_{M} \operatorname{div} \mathrm{T} \mathrm{d} \mathscr{L}^{n}\right| \leqslant \int_{M}|\operatorname{div} \mathrm{T}| \mathrm{d} \mathcal{L}^{n} .
\end{aligned}
$$


In particular, the boundary datum

$$
Q_{1}(S)=\int_{S} \mathrm{Tn}^{B} \mathrm{~d} \mathscr{H}^{n-1}
$$

satisfies (BC) with $v=|\operatorname{div} \mathrm{T}| \mathscr{L}^{n}$. By Theorem 4.1 it follows that $Q_{0}=Q_{1}$.

\section{An Example: Flamant Solution}

In this last section we show how assumption (BC) can be applied to a classical example of linear elasticity: a half-plane with a concentrated load applied perpendicularly to its boundary. If the half-plane is the set $\left\{(x, y) \in \mathbb{R}^{2}: x \geqslant 0\right\}$ and the load $\boldsymbol{p}=p \boldsymbol{e}_{1}$ is applied at the origin $O$ of the frame of reference, then the stress distribution turns out to be (cf. [5, Section 8.3])

$$
\mathrm{T}(x, y)=-\frac{2 p x}{\pi\left(x^{2}+y^{2}\right)^{2}}\left[\begin{array}{cc}
x^{2} & x y \\
x y & y^{2}
\end{array}\right] .
$$

We want to show that the Cauchy flux induced by the stress tensor $T$ admits as boundary datum $Q_{0}$ the vector Dirac delta measure $p \boldsymbol{e}_{1} \delta_{O}$. Note that there is not a classical way to formulate a boundary condition in this setting, since $T$ does not admit a trace in the classical sense. However, by means of Theorem 4.1, we can assert that $p \boldsymbol{e}_{1} \delta_{O}$ is the trace of $\boldsymbol{T n}$ (in a weak sense).

Since our theory strictly applies to bounded bodies, one can cut off from the half-plane a square $(0,2 K) \times(-K, K), K>0$, assigning the value $T n$ to the applied load on the part of boundary of the square which does not lie on the $x$ axis. This does not affect our conclusions.

Consider now the full grid

$$
G=\left(O,\left(\boldsymbol{e}_{1}, \boldsymbol{e}_{2}\right), \mathbb{R} \backslash\{0\}\right),
$$

that is, all the horizontal and vertical lines except the two reference axes. We show that (BC) holds for every $G$-interval (then by additivity it holds for every $G$-figure). Clearly, the key point is to consider intervals of the form $M=(0, a) \times(b, c)$ with $a, c>0$ and $b<0$. It is easy to check that $Q_{0}(\operatorname{bd} M \cap \operatorname{bd} B)=Q_{0}(\{0\} \times$ $\left.(b, c),-\boldsymbol{e}_{1}\right)=p \boldsymbol{e}_{1}$. On the other hand, $Q(B \cap \operatorname{bd} M)=Q\left((0, a) \times\{b\},-\boldsymbol{e}_{2}\right)+$ $Q\left(\{a\} \times(b, c), \boldsymbol{e}_{1}\right)+Q\left((0, a) \times\{c\}, \boldsymbol{e}_{2}\right)$. By straightforward calculations we have that:

$$
\begin{aligned}
Q\left((0, a) \times\{b\},-\boldsymbol{e}_{2}\right) & =\int_{0}^{a} \mathrm{~T}(x, b)\left(-\boldsymbol{e}_{2}\right) \mathrm{d} x \\
& =\frac{p}{\pi}\left[\begin{array}{c}
\arctan \frac{a}{b}-\frac{a b}{a^{2}+b^{2}} \\
\frac{a^{2}}{a^{2}+b^{2}}
\end{array}\right],
\end{aligned}
$$




$$
\begin{aligned}
Q\left(\{a\} \times(b, c), \boldsymbol{e}_{1}\right) & =\int_{b}^{c} \mathrm{~T}(a, y) \boldsymbol{e}_{1} \mathrm{~d} y \\
& =\frac{p}{\pi}\left[\begin{array}{c}
\left.\arctan \frac{b}{a}+\frac{a b}{a^{2}+b^{2}}-\arctan \frac{c}{a}-\frac{a c}{a^{2}+c^{2}}\right], \\
\frac{a^{2}}{a^{2}+c^{2}}-\frac{a^{2}}{a^{2}+b^{2}}
\end{array}\right] \\
Q\left((0, a) \times\{c\}, \boldsymbol{e}_{2}\right) & =\int_{0}^{a} \mathrm{~T}(x, c) \boldsymbol{e}_{2} \mathrm{~d} x \\
& =\frac{p}{\pi}\left[\begin{array}{c}
\frac{a c}{a^{2}+c^{2}}-\arctan \frac{a}{c} \\
-\frac{a^{2}}{a^{2}+c^{2}}
\end{array}\right] .
\end{aligned}
$$

Keeping into account the well-known identity

$$
\arctan x+\arctan \frac{1}{x}= \begin{cases}\frac{\pi}{2} & \text { for } x>0, \\ -\frac{\pi}{2} & \text { for } x<0,\end{cases}
$$

we get immediately $Q(B \cap$ bd $M)=-p \boldsymbol{e}_{1}$. Hence, the boundary condition (BC) is satisfied, for any $M \in \mathcal{M}_{G}$, by choosing $v=0$. Note that for rectangles with a vertex in $O$ the previous calculation fails, because the stress tensor field is not defined at the origin.

\section{References}

1. C. Banfi and M. Fabrizio, Sul concetto di sottocorpo nella meccanica dei continui. Rend. Acc. Naz. Lincei LXVI (1979) 136-142.

2. M. Degiovanni, A. Marzocchi and A. Musesti, Cauchy fluxes associated with tensor fields having divergence measure. Arch. Rational Mech. Anal. 147 (1999) 197-223.

3. M.E. Gurtin and L.C. Martins, Cauchy's theorem in classical physics. Arch. Rational Mech. Anal. 60 (1976) 306-324.

4. M.E. Gurtin, V.J. Mizel and W.O. Williams, A note on Cauchy's stress theorem. J. Math. Anal. Appl. 22 (1968) 398-401.

5. L.E. Malvern, Introduction to the Mechanics of a Continuous Medium. Prentice-Hall, New Jersey (1969).

6. A. Marzocchi and A. Musesti, Decomposition and integral representation of Cauchy interactions associated with measures. Cont. Mech. Thermodyn. 13 (2001) 149-169.

7. W. Noll, The foundations of classical mechanics in the light of recent advances in continuum mechanics. In: The Axiomatic Method, with Special Reference to Geometry and Physics (Berkeley, 1957/58), Studies in Logic and the Foundations of Mathematics, North-Holland, Amsterdam (1959) pp. 266-281.

8. W. Noll and E.G. Virga, Fit regions and functions of bounded variation. Arch. Rational Mech. Anal. 102 (1988) 1-21.

9. P. Podio-Guidugli, Elasticity with live loads. In: Atti dei Convegni Lincei 148 (Roma, 1999) pp. 127-142.

10. M. Šilhavý, The existence of the flux vector and the divergence theorem for general Cauchy fluxes. Arch. Rational Mech. Anal. 90 (1985) 195-211.

11. M. Šilhavý, Cauchy's stress theorem and tensor fields with divergences in $L^{p}$. Arch. Rational Mech. Anal. 116 (1991) 223-255. 